Masaru Takeuchi

Nagoya Math. J.

Vol. 40 (1970), 147-159

\title{
ON THE FUNDAMENTAL GROUP OF A SIMPLE LIE GROUP
}

\author{
MASARU TAKEUCHI
}

\section{Introduction}

Let $G$ be a simply connected simple Lie group and $C$ the center of $G$, which is isomorphic with the fundamental group of the adjoint group of $G$. For an element $c$ of $C$, an element $x$ of the Lie algebra $g$ of $G$ is called a representative of $c$ in $g$ if $\exp x=c$. Sirota-Solodovnikov [7] found a complete set of representatives of the center $C$ in $g$ and studied the group structure of $C$, and using their results Goto-Kobayashi [1] classified subgroups of the center $C$ with respect to automorphisms of $G$. The group structure of $C$ was also studied in Takeuchi [8].

Sirota-Solodovnikov's complete representatives were obtained by calculating a free abelian group $Z_{*}$ modulo a subgroup $Z_{0}$ for each simple group. But if $G$ is compact, owing to the classical result of E. Cartan, they are obtained systematically as follows. Let $\mathfrak{h}$ be a maximal abelian subalgebra of $\mathrm{g}, \Delta$ the root system of the complexification $\mathrm{g}^{c}$ of $\mathrm{g}$ with respect to the complexification $\mathfrak{h}^{c}$ of $\mathfrak{h}$ and $\Pi=\left\{\alpha_{1}, \cdots, \alpha_{l}\right\}$ a fundamental system of $\Delta$. Let $\mu=\sum_{i=1}^{l} n_{i} \alpha_{i}$ be the highest root of $\Delta$ with respect to $\Pi$ and $\Lambda_{i}^{*}(1 \leqslant i \leqslant l)$ the dual basis of $\Pi$ in $\sqrt{-1} \mathfrak{h}$ defined by the relations: $\alpha_{i}\left(\Lambda_{j}^{*}\right)=\delta_{i j}(1 \leqslant i$, $j \leqslant l$ ). Then the set $\{0\} \cup\left\{2 \pi \sqrt{-1} \Lambda_{i}^{*} ; 1 \leqslant i \leqslant l, n_{i}=1\right\}$ give a complete set of representatives of the center $C$ in $g$. Thus it is quite easy to see how an automorphism of $G$ acts on $C$.

Moreover, by an unpublished result of Murakami (Theorem 2), if $G$ is compact, $C$ is isomorphic with a subgroup of the group of automorphisms of the extended fundamental system $\Pi^{*}$, where $\Pi^{*}$ is defined from $\Pi$ by adding $-\mu$ to it.

In this note we shall generalize the above results to general $G$ (not necessarily compact). A complete set of representatives of the center $C$ in

Received June 5, 1969. 
$\mathrm{g}$ is obtained by seeing the fundamental system and the highest root of $\mathrm{g}$ and those of simple components of a maximal compact subalgebra $\mathfrak{k}$ of in terms of the dual basis of fundamental systems. The group strucutre of the center $C$ is described by means of a group of automorphisms of the "extended fundamental system" of $\mathfrak{k}$. Thus it is immediate to find the action of automorphisms of $G$ on $C$.

\section{§1. Fundamental group of a semi-simple group}

Let $G$ be a connected semi-simple Lie group and $\mathfrak{g}$ its Lie algebra. Let $\mathfrak{f}$ be a maximal compact subalgebra of $\mathfrak{g}$, $\mathfrak{f}^{\prime}$ the derived algebra of $\mathfrak{f}$ and $K$ (resp. $K^{\prime}$ ) the connected subgroup of $G$ generated by $\mathfrak{l}^{\prime}$ resp. $\left.\mathfrak{l}^{\prime}\right)$. Then we have $\pi_{1}(K) \cong \pi_{1}(G)$ since $G$ is diffeomorphic with the product of $K$ and a Euclidean space (Helgason [22], p. 214). We take a Cartan subalgebra $\mathfrak{h}_{1}$ of $\mathfrak{f}$. Then $\mathfrak{h}_{1}$ contains a regular element of $\mathfrak{g}$ (Murakami [5]) so that $\mathfrak{h}_{1}$ can be extended uniquely to a Cartan subalgebra $\mathfrak{h}$ of $\mathfrak{g}$. Let $\mathfrak{g}^{c}$ (resp. $\mathfrak{f}^{c}, \mathfrak{f}^{\prime} C, \mathfrak{h}^{c}$ ) denote the complexification of $\mathfrak{g}$ (resp. $\left.\mathfrak{f}, \mathfrak{f}^{\prime}, \mathfrak{h}\right)$ and let $\mathfrak{h}_{+}^{C}=\mathfrak{h}^{c} \cap \mathfrak{F}$, $\mathfrak{h}^{\prime} C=\mathfrak{h}^{C} \cap \mathfrak{l}^{\prime} C$. Then $\mathfrak{h}^{C}$ (resp. $\mathfrak{h}_{+}^{C}, \mathfrak{h}^{\prime} C$ ) is a Cartan subalgebra of $\mathfrak{g}^{C}$ (resp. $\mathfrak{l}^{C}, \mathfrak{f}^{\prime} C$ ). Let $\mathfrak{h}_{0}$ be the real part of $\mathfrak{h}^{c}$ and put $\mathfrak{h}_{+}=\mathfrak{h}_{0} \cap \mathfrak{h}_{+}^{c}, \mathfrak{h}^{\prime}=\mathfrak{h}_{0} \cap \mathfrak{h}^{\prime} c, c=$ the orthogonal complement of $\mathfrak{h}^{\prime}$ in $\mathfrak{h}_{+}$with respect to the Killing form (, ) of $\mathfrak{g}^{c}$. Then the Weyl group $W\left(\operatorname{resp} . W^{\prime}\right)$ of $\mathfrak{l}^{C}\left(\operatorname{resp} . \mathfrak{f}^{\prime} \boldsymbol{c}\right)$ on $\mathfrak{h}_{+}^{c}\left(\operatorname{resp} \cdot \mathfrak{h}^{\prime} \boldsymbol{C}\right)$ is considered as a group of orthogonal transformations of $\mathfrak{h}_{+}\left(\right.$resp. $\left.\mathfrak{h}^{\prime}\right)$ with respect to the Killing form of $\mathfrak{g}^{c}$ and $W$ acts trivially on $\mathfrak{c}$ and coincides with $W^{\prime}$ on $\mathfrak{h}^{\prime}$. In the following we shall identify the dual space of $\mathfrak{h}_{0}$ with $\mathfrak{h}_{0}$ by means of the Killing form of $\mathrm{g}^{c}$, so that the root system $\Delta$ (resp. $\Delta^{\prime}$ ) of $\mathfrak{g}^{C}$ (resp. $\mathfrak{f}^{\prime} C$ ) with respect to $\mathfrak{h}^{C}\left(\right.$ resp. $\mathfrak{h}^{\prime} C$ ) is contained in $\mathfrak{h}_{0}$ (resp. $\mathfrak{h}^{\prime}$ ). Let $\Pi^{\prime}=\left\{\beta_{1}, \cdots, \beta_{l^{\prime}}\right\}$ be a fundamental system of $\Delta^{\prime}$ and $>$ the lexicographic order of $\Delta^{\prime}$ associated with $\Pi^{\prime}$. Now we put

$$
Z=\frac{1}{2 \pi \sqrt{-1}} \text { kernel }\left\{\exp : \sqrt{-1} \mathfrak{h}_{+} \longrightarrow K\right\}
$$

and let $t(z)$ denote the translation $h \longmapsto h+z$ of $\mathfrak{h}_{+}$by an element $z$ of $Z$. Then $W \cap t(Z)=\{1\}$ and $W$ normalizes $t(Z)$ since $W$ leaves $Z$ invariant and $w t(z) w^{-1}=t(w z)$ for $w \in W$ and $z \in Z$. Thus we have a group $\tilde{W}$ of isometries of the Euclidean space $\mathfrak{h}_{+}$defining that

$$
\tilde{W}=t(Z) W
$$

The groups $Z$ and $\tilde{W}$ for the universal covering group of $G$ or the adjoint 
group of $G$ will be denoted by $Z_{0}$ and $\tilde{W}_{0}$ or $Z_{*}$ and $\tilde{W}_{*}$. Then we have

$$
\begin{aligned}
& Z_{*}=\left\{h \in \mathfrak{h}_{+} ;(\alpha, h) \in \boldsymbol{Z} \text { for any root } \alpha \text { of } \Delta\right\}, \\
& Z_{0}=\sum_{i=1}^{l^{\prime}} \boldsymbol{Z} \beta_{i}^{*}, \text { where } \beta_{i}^{*}=\left(2 /\left(\beta_{i}, \beta_{i}\right)\right) \beta_{i} .
\end{aligned}
$$

The latter equality follows from the fact that the righthand side is the dual group of the group of weights of $\mathfrak{f}^{\prime} c$. It is clear that $Z_{0} \subset Z \subset Z_{*}$ and $\tilde{W}_{0} \subset \tilde{W} \subset \tilde{W}_{*}$. If we denote by $K_{0}$ the simply connected subgroup of the

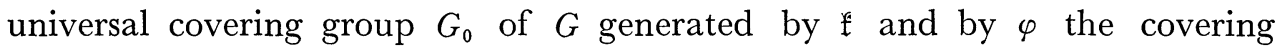
homomorphism of $K_{0}$ onto $K$, then the map $\gamma: Z \mid Z_{0} \longrightarrow G_{0}$ defined by $z \bmod Z_{0} \mid \exp _{G_{0}} 2 \pi \sqrt{-1} z$ induces the isomorphism of $Z / Z_{0}$ onto the kernel of $\varphi$, which is isomorphic with $\pi_{1}(K) \cong \pi_{1}(G)$. Thus

$$
Z / Z_{0} \cong \pi_{1}(G)
$$

Lemma 1. $w z \equiv z\left(\bmod Z_{0}\right)$ for $w \in W$ and $z \in Z$.

Proof. There exists an element $k$ of the normalizer in $K_{0}$ of $\mathfrak{h}_{+}$such that $\operatorname{Ad} k$ restricted to $\mathfrak{h}_{+}$coincides with $w$. Since the kernel of the above covering homomorphism $\varphi$ is contained in the center of $K_{0}$, the element $k$ centralizes the kernel of $\varphi$, which yields Lemma. $\quad$ q.e.d.

Note that $(z, \beta) \in \boldsymbol{Z}$ for $z \in Z$ and $\beta \in \Delta^{\prime}$, since $\beta$ is obtained as the orthogonal projection to $\mathfrak{h}_{+}$of some root of $\Delta$ and $Z \subset Z_{*}$. This fact will be used sometimes in the following.

The subset

$$
D=\left\{h \in \mathfrak{h}_{+} ;(h, \beta) \in \boldsymbol{Z} \text { for some root } \beta \text { of } \Delta^{\prime}\right\}
$$

of $\mathfrak{h}_{+}$is called the diagram of $\mathfrak{x}$ on $\mathfrak{h}_{+}$and a connected component of $\mathfrak{h}_{+}-D$ is called a cell of $\mathfrak{f}$ on $\mathfrak{h}_{+}$. Then $\tilde{W}$ leaves $D$ invariant since $W\left(\Delta^{\prime}\right) \subset \Delta^{\prime}$ and $\left(Z, \Delta^{\prime}\right) \subset \boldsymbol{Z}$. It follows that $\tilde{W}$ acts on the set of cells of $\mathfrak{t}$ on $\mathfrak{H}_{+}$. A classical theorem of E. Cartan (cf. Helgason [2], p. 265) says that $\tilde{W}_{0}$ acts simply transitively on the set of cells. (An algebraic proof of this theorem is seen in Iwahori-Matsumoto [3].) Let $\mathscr{C}^{\prime}$ be the positive Weyl chamber of $\mathfrak{f}^{\prime}$ on $\mathfrak{h}^{\prime}$ with respect to $\Pi^{\prime}$, that is, $\mathscr{C}^{\prime}=\left\{h \in \mathfrak{h}^{\prime} ;\left(h, \beta_{i}\right)>0\right.$ for any root $\beta_{i}$ of $\left.\Pi^{\prime}\right\}$, and $S$ the unique cell of in $\mathfrak{h}_{+}$such that the closure $\bar{S}$ of $S$ contains 0 and $S \cap \mathscr{C}^{\prime} \neq \phi$. We put

$$
\tilde{W}(S)=\{\tau \in \tilde{W} ; \tau S=S\} .
$$


THEOREM 1. The group $\tilde{W}(S)$ is isomorphic with the fundamental group $\pi_{1}(G)$ of $G$.

Proof. Let us consider the map of $\tilde{W}$ to $Z \mid Z_{0}$ defined by $t(z) w \mid \longrightarrow$ $z \bmod Z_{0}$ for $z \in Z$ and $w \in W$. Since we have $\left(t\left(z_{1}\right) w_{1}\right)\left(t\left(z_{2}\right) w_{2}\right)=t\left(z_{1}+w_{1} z_{2}\right)\left(w_{1} w_{2}\right)$, the map is a homomorphism in view of Lemma 1 . The kernel of this homomorphism is just the group $\tilde{W}_{0}$. It follows that $\tilde{W}_{0}$ is a normal subgroup of $\tilde{W}$ and $\tilde{W} / \tilde{W}_{0} \cong Z / Z_{0} \cong \pi_{1}(G)$. On the other hand, the theorem of E. Cartan yields that $\tilde{W}$ is the semi-direct product of $\tilde{W}(S)$ and $\tilde{W}_{0}$. It follows that $\tilde{W}(S) \cong \tilde{W} / \tilde{W}_{0} \cong \pi_{1}(G)$.

q.e.d.

CoRollary. The corresponding group $\tilde{W}_{*}(S)$ for centerless group $G$ is isomorphic with the center $C$ of the universal covering group of $G$.

Proof. Obvious since $\pi_{1}(G)$ is isomorphic with $C$. An explicit isomorphism is given by $\tilde{W}_{*}(S) \cong Z_{*} \mid Z_{0} \stackrel{\gamma}{\cong} C$.

q.e.d.

REMARK. The group $\tilde{W}(S)$ may be described in terms of covering transformations of the universal covering space of an open submanifold of $K$ (cf. Takeuchi [8], Helgason [2]).

If we put $S^{\prime}=\mathfrak{h}^{\prime} \cap S$, we have $S=\mathfrak{c} \times S^{\prime}$. Now we define certain groups on $\mathfrak{h}^{\prime}$ similarly to those on $\mathfrak{h}_{+}$. Let

$$
\begin{aligned}
& Z_{*}^{\prime}=\left\{h \in \mathfrak{h}^{\prime} ;(h, \beta) \in Z \text { for any root } \beta \text { of } \Delta^{\prime}\right\}, \\
& \tilde{W}_{*}^{\prime}=t^{\prime}\left(Z_{*}^{\prime}\right) W^{\prime}, \text { where } t^{\prime}\left(z^{\prime}\right) h^{\prime}=z^{\prime}+h^{\prime} \text { for } h^{\prime} \in \mathfrak{h}^{\prime} .
\end{aligned}
$$

Then $Z_{*}^{\prime}$ contains $Z \cap \mathfrak{H}^{\prime}$ and $\tilde{W}_{*}^{\prime}$ leaves $D^{\prime}=\mathfrak{h}^{\prime} \cap D$ invariant so that $\tilde{W}_{*}^{\prime}$ acts on connected components of $\mathfrak{h}^{\prime}-D^{\prime}$, which are called cells of $\mathfrak{f}^{\prime}$ on $\mathfrak{h}^{\prime}$. $S^{\prime}$ is the unique cell of $\mathfrak{l}^{\prime}$ on $\mathfrak{h}^{\prime}$ such that $\bar{S}^{\prime}$ contains 0 and $S^{\prime} \cap \mathscr{C}^{\prime} \neq \phi$. Put

$$
\tilde{W}_{*}^{\prime}\left(S^{\prime}\right)=\left\{\tau^{\prime} \in \tilde{W}_{*}^{\prime} ; \tau^{\prime} S^{\prime}=S^{\prime}\right\}
$$

The same argument as above shows that $\tilde{W}_{*}^{\prime}\left(S^{\prime}\right)$ is isomorphic with the fundamental group of the adjoint group of $\mathfrak{f}^{\prime}$ and with the center of the universal covering group of $K^{\prime}$.

Lemma 2. 1) Let $Z^{\prime \prime}$ be the image of $\bar{S} \cap Z$ by the orthogonal projection of $\mathfrak{H}_{+}$onto c. Then $\bar{S} \cap Z \subset Z^{\prime \prime} \times\left(\bar{S}^{\prime} \cap Z_{*}^{\prime}\right)$.

2) Let $\xi(\tau)=\tau(0)$ for $\tau \in \tilde{W}(S)$. Then the map $\xi$ gives a bijection of $\tilde{W}(S)$ 
onto $\bar{S} \cap Z$. The set $2 \pi \sqrt{-1}\left(\bar{S} \cap Z_{*}\right)$ is a complete set of representatives in $\mathrm{g}$ of the center $C$ of the universal covering group of $G$.

3) Let $\xi^{\prime}\left(\tau^{\prime}\right)=\tau^{\prime}(0)$ for $\tau^{\prime} \in \tilde{W}_{*}^{\prime}\left(S^{\prime}\right)$. Then the map $\xi^{\prime}$ gives a bijection of $\tilde{W}_{*}^{\prime}\left(S^{\prime}\right)$ onto $\bar{S}^{\prime} \cap Z_{*}^{\prime}$. The set $2 \pi \sqrt{-1}\left(\bar{S}^{\prime} \cap Z_{*}^{\prime}\right)$ is a complete set of representatives in $\mathfrak{I}^{\prime}$ of the center of the universal covering group of $K^{\prime}$.

Proof. 1) Let $z=\left(z^{\prime \prime}, z^{\prime}\right)$ be an element of $\bar{S} \cap Z=\left(\mathfrak{c} \times \bar{S}^{\prime}\right) \cap Z$, where $z^{\prime \prime} \in Z^{\prime \prime}$ and $z^{\prime} \in \bar{S}^{\prime}$. Then for any $\operatorname{root} \beta$ of $\Delta^{\prime}$ we have $\left(z^{\prime}, \beta\right)=(z, \beta)-$ $\left(z^{\prime \prime}, \beta\right)=(z, \beta) \in \boldsymbol{Z}$ so that $z^{\prime} \in \bar{S}^{\prime} \cap Z_{*}^{\prime}$.

2) For any element $\tau=t(z) w$ of $\tilde{W}(S)$, where $z \in Z$ and $w \in W$, we have $\xi(\tau)=\tau(0)=z \in Z$. It follows that $\xi(\tau) \in \bar{S} \cap Z$ since $0 \in \bar{S}$. We shall show first that $\xi$ is surjective. In view of 1 ), any element $z$ of $\bar{S} \cap Z$ can be written as $z=z^{\prime \prime}+z^{\prime}$, where $z^{\prime \prime} \in Z^{\prime \prime}$ and $z^{\prime} \in \bar{S}^{\prime} \cap Z_{*}^{\prime}$. Then $t(z)^{-1} S=$ $\mathfrak{c} \times t\left(z^{\prime}\right)^{-1} S^{\prime}$ and $t\left(z^{\prime}\right)^{-1} S^{\prime}$ is a cell of $\mathfrak{f}^{\prime}$ on $\mathfrak{h}^{\prime}$ such that its closure contains 0 . Since $W^{\prime}$ acts transitively on Weyl chambers of $\mathfrak{f}^{\prime}$ on $\mathfrak{h}^{\prime}$, we have an element $w$ of $W$ such that $w^{-1} t\left(z^{\prime}\right)^{-1} S^{\prime}=S^{\prime}$. It follows that $w^{-1} t(z)^{-1} S=$ $\mathfrak{c} \times S^{\prime}=S$ so that $\tau=t(z) w \in \tilde{W}(S)$ and $\xi(\tau)=z$. We shall show next that $\xi$ is injective. Let $\tau_{i}=t\left(z_{i}\right) w_{i}(i=1,2)$ be elements of $\tilde{W}(S)$ such that $\xi\left(\tau_{1}\right)=$ $\xi\left(\tau_{2}\right)$. Then we have $z_{1}=z_{2}$ and $\tau_{2}^{-1} \tau_{1}=w_{2}^{-1} w_{1} \in W \cap \tilde{W}(S) \subset \tilde{W}_{0} \cap \tilde{W}(S)$. But since $\tilde{W}_{0} \cap \tilde{W}(S)=\{1\}$ by the theorem of E. Cartan, we have $\tau_{1}=\tau_{2}$. The second statement follows from the first statement and Corollary of Theorem 1.

3) is proved similarly to the above.

q.e.d.

Lemma 3. 1) $Z^{\prime \prime}$ is a subgroup of c. The corresponding group $Z_{*}^{\prime \prime}$ for centerless group $G$ is a lattice of $\mathfrak{c}$.

2) Let $F$ be the subset of $\tilde{W}_{*}^{\prime}\left(S^{\prime}\right)$ corresponding to $\bar{S}^{\prime} \cap Z$ under the bijection $\xi^{\prime}: \tilde{W}_{*}^{\prime}\left(S^{\prime}\right) \longrightarrow \bar{S}^{\prime} \cap Z_{*}^{\prime}$ and let $\pi^{\prime \prime}(\tau)=z^{\prime \prime}$ for an element $\tau=t\left(z^{\prime \prime}+z^{\prime}\right) w$ of $\tilde{W}(S)$, where $z^{\prime \prime} \in Z^{\prime \prime}, z^{\prime} \in \bar{S}^{\prime} \cap Z_{*}^{\prime}$ and $w \in W$. Then $F$ is a subgroup of $\tilde{W}_{*}^{\prime}\left(S^{\prime}\right)$ and the map $\pi^{\prime \prime}: \tilde{W}(S) \longrightarrow Z^{\prime \prime}$ is a homomorphism. Moreover we have a split exact sequence:

$$
0 \longrightarrow F \longrightarrow \tilde{W}(S) \stackrel{\pi^{\prime \prime}}{\longrightarrow} Z^{\prime \prime} \longrightarrow 0 .
$$

Thus we have an isomorphism: $\tilde{W}(S) \cong Z^{\prime \prime} \times F$.

Proof. For elements $\tau_{i}=t\left(z_{\imath}^{\prime \prime}+z_{i}^{\prime}\right) w_{i}$ of $\tilde{W}(S)(i=1,2)$, we have $\tau_{1} \tau_{2}=$ $t\left(\left(z_{1}^{\prime \prime}+z_{2}^{\prime \prime}\right)+\left(z_{1}^{\prime}+w_{1} z_{2}^{\prime}\right)\right)\left(w_{1} w_{2}\right)$ so that $\pi^{\prime \prime}$ is a homomorphism of $\tilde{W}(S)$ into $c$. Since $\pi^{\prime \prime} \tilde{W}=Z^{\prime \prime}$ in view of Lemma 2, $Z^{\prime \prime}$ is a subgroup of $\mathfrak{c}$. 
If $\pi^{\prime \prime}(\tau)=0$ for an element $\tau=t(z) w$ of $\tilde{W}(S)$, then $z \in \mathfrak{h}^{\prime} \cap Z \subset Z_{*}^{\prime}$. It follows that $\tau$ is identity on $\mathfrak{c}$, its restriction $\tau^{\prime}$ to $\mathfrak{h}^{\prime}$ belongs to $\tilde{W}_{*}^{\prime}\left(S^{\prime}\right)$ and $\xi^{\prime}\left(\tau^{\prime}\right) \in \bar{S}^{\prime} \cap Z$. Conversely if $\tau^{\prime}$ is an element of $\tilde{W}_{*}^{\prime}\left(S^{\prime}\right)$ with $\xi^{\prime}\left(\tau^{\prime}\right) \in \bar{S}^{\prime} \cap Z$, then the trivial extension $\tau$ of $\tau^{\prime}$ to $\mathfrak{h}_{+}$satisfies $\tau \in \tilde{W}(S)$ and $\tilde{w}^{\prime \prime}(\tau)=0$. It follows that $F$ is a subgroup of $\tilde{W}_{*}^{\prime}\left(S^{\prime}\right)$ and isomorphic with the kernel of $\pi^{\prime \prime}$. So we have the desired exact sequence, which splits because $Z^{\prime \prime}$ is free.

If $G$ is centerless, then $K$ is compact so that $Z_{*} \cap \mathfrak{c}$ is a lattice of $\mathrm{c}$. Since $Z_{*}^{\prime \prime}$ contains $Z_{*} \cap \mathfrak{c}, Z_{*}^{\prime \prime}$ is also a lattice of $\mathfrak{c}$ q.e.d.

Now we want to describe the structure of the group $F$. Let $\mathfrak{x}^{\prime}=\sum_{i=1}^{r} \mathfrak{f}_{i}^{\prime}$ be the decomposition of $\mathfrak{f}^{\prime}$ into simple factors. Then $\mathfrak{h}^{\prime}, \Delta^{\prime}, \Pi^{\prime}, Z_{*}^{\prime}, S^{\prime}, \bar{S}^{\prime} \cap Z_{*}^{\prime}$, $W^{\prime}, \tilde{W}_{*}^{\prime}$ and $\tilde{W}_{*}^{\prime}\left(S^{\prime}\right)$ are the direct products of corresponding objects for simple factors $\mathfrak{f}_{i}$, which will be denoted by the same symbol with the suffix $i$. Let $\mu_{i}^{\prime}$ be the highest root of $\Delta_{i}^{\prime}$ and $\Pi_{i}^{\prime *}=\Pi_{i}^{\prime} \cup\left\{-\mu_{i}^{\prime}\right\}$. Let $\operatorname{Aut}\left(\Pi_{i}^{\prime *}\right)$ denote the group of orthogonal transformations of $\mathfrak{h}_{i}^{\prime}$ preserving $\Pi_{i}^{\prime *}$ and let

$$
\begin{aligned}
& \Pi^{\prime *}=\bigcup_{i=1}^{r} \Pi_{i}^{\prime *}, \\
& \operatorname{Aut}\left(\Pi^{\prime *}\right)=\prod_{i=1}^{r} \operatorname{Aut}\left(\Pi_{i}^{\prime *}\right) .
\end{aligned}
$$

Theorem 2. 1) Let $\pi^{\prime}\left(\tau^{\prime}\right)=w^{\prime}$ for an element $\tau^{\prime}=t^{\prime}\left(z^{\prime}\right) w^{\prime}$ of $\tilde{W}_{*}^{\prime}\left(S^{\prime}\right)$, where $z^{\prime} \in Z_{*}^{\prime}$ and $w^{\prime} \in W^{\prime}$. Then $\pi^{\prime}\left(\tau^{\prime}\right) \in$ Aut $\left(\Pi^{*}\right)$ for any element $\tau^{\prime}$ of $\tilde{W}_{*}^{\prime}\left(S^{\prime}\right)$ and the map $\pi^{\prime}: \tilde{W}_{*}^{\prime}\left(S^{\prime}\right) \longrightarrow$ Aut $\left(\Pi^{\prime *}\right)$ is an injective homomorphism. The image $\pi^{\prime} \tilde{W}_{*}^{\prime}\left(S^{\prime}\right)$ of $\pi^{\prime}$ will be denoted by $\mathscr{F}\left(\mathfrak{f}^{\prime}\right)$, which is isomorphic with the fundamental group of the adjoint group of $\mathfrak{l}^{\prime}$.

2) If $\mathfrak{f}^{\prime}$ is simple, the group $\mathscr{F}\left(\mathfrak{f}^{\prime}\right)$ is obtained as follows. Let $M_{i}^{*} \in \mathfrak{h}^{\prime}$ $\left(1 \leqslant i \leqslant l^{\prime}\right)$ be the dual basis of $\Pi^{\prime}$, that is, $\left(M_{i}^{*}, \beta_{j}\right)=\delta_{i j}\left(1 \leqslant i, j \leqslant l^{\prime}\right)$ and $P_{i}=\left(1 / m_{i}\right) M_{i}^{*}\left(1 \leqslant i \leqslant l^{\prime}\right)$, where $m_{i}$ is the $i$-th coefficient of the highest root $\mu^{\prime}=\sum_{i=1}^{l^{\prime}} m_{i} \beta_{i}$ of $\Delta^{\prime}$. We put $\beta_{0}=-\mu^{\prime}, M_{0}^{*}=P_{0}=0$ and $m_{0}=1$. Then

a) $\left\{P_{0}, P_{1}, \cdots, P_{l^{\prime}}\right\}$ is the set of vertices of $\bar{S}^{\prime}$.

b) $\bar{S}^{\prime} \cap Z_{*}^{\prime}=\left\{M_{i}^{*} ; 0 \leqslant i \leqslant l^{\prime}, m_{i}=1\right\}$ and the set $\left\{2 \pi \sqrt{-1} M_{i}^{*} ; 0 \leqslant i \leqslant l^{\prime}\right.$, $\left.m_{i}=1\right\}$ is a complete set of representatives of the center of the simply connected Lie group with the Lie algebra $\mathfrak{\mathfrak { H }}^{\prime}$.

c) Let $\tau_{i}^{\prime}$ be the element of $\tilde{W}_{*}^{\prime}\left(S^{\prime}\right)$ with $\xi^{\prime}\left(\tau_{i}^{\prime}\right)=M_{i}^{*}$ and $\pi_{i}$ the element of the symmetric group of $\left(l^{\prime}+1\right)$ letters $\left\{0,1, \cdots, l^{\prime}\right\}$ defined by $\tau_{i}^{\prime} P_{j}=P_{\pi_{i}(j)}\left(0 \leqslant j \leqslant l^{\prime}\right)$. Then $\pi^{\prime}\left(\tau_{i}^{\prime}\right) \beta_{j}=\beta_{\pi_{i}(j)}\left(0 \leqslant j \leqslant l^{\prime}\right)$. 
d) $\pi^{\prime}\left(\tau_{i}^{\prime}\right)$ is characterized by the property:

$$
\left\{\beta \in \Delta^{\prime} ; \beta>0, \pi^{\prime}\left(\tau_{i}^{\prime}\right)^{-1} \beta<0\right\}=\left\{\beta \in \Delta^{\prime} ;\left(\beta, M_{\imath}^{*}\right)>0\right\} .
$$

Proof. They were proved in a more general situation in Takeuchi [8] except 2), d) and the last statement was contained together with the other in Iwahori-Matsumoto [3], but we prove them again here for the sake of completeness.

Since we have $\tau_{1}^{\prime} \tau_{2}^{\prime}=t^{\prime}\left(z_{1}^{\prime}+w_{1}^{\prime} z_{2}^{\prime}\right)\left(w_{1}^{\prime} w_{2}^{\prime}\right)$ for $\tau_{\imath}^{\prime}=t^{\prime}\left(z_{i}^{\prime}\right) w_{i}^{\prime} \in \tilde{W}_{*}^{\prime}\left(S^{\prime}\right)(i=1,2)$, $\pi^{\prime}$ is a homomorphism of $\tilde{W}_{*}^{\prime}\left(S^{\prime}\right)$ to $W^{\prime}$. To prove the statements that $\pi^{\prime} \tilde{W}_{*}^{\prime}(S) \subset$ Aut $\left(\Pi^{\prime *}\right)$ and $\pi^{\prime}$ is injective, we may assume that $\mathfrak{f}^{\prime}$ is simple. But in this case they are true in view of 2), c).

2) a) follows from

$$
\begin{aligned}
& S^{\prime}=\left\{h^{\prime} \in \mathfrak{h}^{\prime} ;\left(h^{\prime}, \beta_{i}\right)>0\left(1 \leqslant i \leqslant l^{\prime}\right), \quad\left(h^{\prime}, \mu^{\prime}\right)<1\right\}, \\
& \bar{S}^{\prime}=\left\{h^{\prime} \in \mathfrak{h}^{\prime} ; \quad\left(h^{\prime}, \beta_{i}\right) \geqslant 0\left(1 \leqslant i \leqslant l^{\prime}\right), \quad\left(h^{\prime}, \mu^{\prime}\right) \leqslant 1\right\} .
\end{aligned}
$$

b) The first statement follows from a) and that $Z_{*}^{\prime}=\sum_{i=1}^{l^{\prime}} \boldsymbol{Z} M_{i}^{*}$. The second follows from Lemma 2, 3).

c) We shall show first that $m_{j}=m_{\pi_{i}(j)}\left(0 \leqslant j \leqslant l^{\prime}\right)$. Since $\pi^{\prime}\left(\tau_{i}^{\prime}\right)=$ $t^{\prime}\left(\xi^{\prime}\left(\tau_{i}^{\prime}\right)\right)^{-1} \tau_{i}^{\prime}$, we have $\pi^{\prime}\left(\tau_{i}^{\prime}\right) P_{j}=P_{\pi_{i}(j)}-\xi^{\prime}\left(\tau_{i}^{\prime}\right)=\left(1 / m_{\pi_{i}(j)}\right) M_{\pi_{l}(j)}{ }^{*}-\xi^{\prime}\left(\tau_{i}^{\prime}\right)$ and therefore

$$
\pi^{\prime}\left(\tau_{i}^{\prime}\right) M_{j}^{*}=\left(m_{j} / m_{\pi_{\imath}(j)}\right) M_{\pi_{\imath}(j)}^{*}-m_{j} \xi^{\prime}\left(\tau_{i}^{\prime}\right)
$$

Hence $\left(m_{j} / m_{\pi_{i}(j)}\right) M_{\pi_{i}(j)}^{*} \in Z_{*}^{\prime}$. It follows from the equality: $Z_{*}^{\prime}=\sum_{k=1}^{l^{\prime}} \boldsymbol{Z} M_{k}^{*}$ that $m_{j} / m_{\pi_{l}(j)} \geqslant 1$. The same argument for $\tau_{\imath}^{-1}$ shows that $m_{\pi_{l}(j)} / m_{j} \geqslant 1$. Thus we have $m_{j}=m_{\pi_{r}(j)}$.

Since $\xi^{\prime}\left(\tau_{i}^{\prime}\right)=\tau_{\imath}^{\prime}(0)=\tau_{i}^{\prime} P_{0}=P_{\pi_{i}(0)}=\left(1 / m_{\pi_{2}(0)}\right) M_{\pi_{i}(0)}^{*}=\left(1 / m_{0}\right) M_{\pi_{i}(0)}^{*}=M_{\pi_{i}(0)}^{*}$, we have from $(*)$ that $\pi^{\prime}\left(\tau_{i}^{\prime}\right) M_{j}^{*}=M_{\pi_{\imath}(j)}^{*}-m_{j} M_{\pi_{i}(0)}^{*}$. Replacing $\tau_{i}$ by $\tau_{\imath}^{-1}$ we have

$$
\pi^{\prime}\left(\tau_{i}^{\prime}\right)^{-1} M_{j}^{*}=M_{\pi_{i}^{-1}(j)}^{*}-m_{j} M_{\pi_{i}^{-1}(0)}^{*}\left(0 \leqslant j \leqslant l^{\prime}\right) .
$$

Now it is easy to derive $\pi^{\prime}\left(\tau_{i}^{\prime}\right) \beta_{j}=\beta_{\pi_{i}(j)}$ using $m_{j}=m_{\pi_{i}(j)}$ and the above equalities: If $j \neq 0, \pi_{\imath}^{-1}(0)$, then for $1 \leqslant k \leqslant l^{\prime}$ we have $\left(\pi^{\prime}\left(\tau_{\imath}^{\prime}\right) \beta_{j}, M_{k}^{*}\right)=$ $\left(\beta_{j}, \pi^{\prime}\left(\tau_{i}^{\prime}\right)^{-1} M_{k}^{*}\right)=\left(\beta_{j}, M_{\pi_{i}^{-1}(k)}^{*}-m_{k} M_{\pi_{i}^{-1}(0)}^{*}\right)=\left(\beta_{j}, M_{\pi_{i}^{-1}(k)}^{*}\right)=\delta_{\pi_{i}(j), k}=\left(\beta_{\pi_{i}(j)}, M_{k}^{*}\right)$, so that $\pi^{\prime}\left(\tau_{i}^{\prime}\right) \beta_{j}=\beta_{\pi_{l}(j)}$. We can similarly confirm the same equality for $j=0$ or $\pi_{i}^{-1}(0)$. 
d) Since the existence and the uniqueness of an element $w^{\prime}$ of $W^{\prime}$ such that

$$
\left\{\beta \in \Delta^{\prime} ; \beta>0, w^{\prime-1} \beta<0\right\}=\left\{\beta \in \Delta^{\prime} ;\left(\beta, M_{i}^{*}\right)>0\right\}
$$

is known (Kostant [4]), it suffices to show that $\tau^{\prime}=t^{\prime}\left(M_{i}^{*}\right) w^{\prime}$, with $w^{\prime}$ as above and $m_{i}=1$, leaves $S^{\prime}$ invariant. We may assume that $i \neq 0$. Take an element $h^{\prime}$ of $S^{\prime}$. Let $1 \leqslant j \leqslant l^{\prime}$, then $\left(\tau^{\prime} h^{\prime}, \beta_{j}\right)=\left(w^{\prime} h^{\prime}+M_{i}^{*}, \beta_{j}\right)=$ $\left(h^{\prime}, w^{\prime-1} \beta_{j}\right)+\left(M_{i}^{*}, \beta_{j}\right)$. If $w^{\prime-1} \beta_{j}>0$, then $\left(h^{\prime}, w^{\prime-1} \beta_{j}\right)>0$ since $h^{\prime} \in S^{\prime}$. If $w^{\prime-1} \beta_{j}<0$, then $\left(M_{i}^{*}, \beta_{j}\right)=1$ from the assumption for $w^{\prime}$ and $\left(h^{\prime}, w^{\prime-1} \beta_{j}\right)>-1$ since $h^{\prime} \in S^{\prime}$. Thus in both cases we have $\left(\tau^{\prime} h^{\prime}, \beta_{j}\right)>0$. Furthermore we have $\left(\tau^{\prime} h^{\prime}, \mu^{\prime}\right)=\left(w^{\prime} h^{\prime}+M_{i}^{*}, \mu^{\prime}\right)=\left(h^{\prime}, w^{\prime-1} \mu^{\prime}\right)+1$. If $w^{\prime-1} \mu^{\prime}<0$, then $\left(h^{\prime}, w^{\prime-1} \mu^{\prime}\right)<0$ since $h^{\prime} \in S^{\prime}$, so that $\left(\tau^{\prime} h^{\prime}, \mu^{\prime}\right)<1$. If $w^{\prime-1} \mu^{\prime}>0$, then from the assumption for $w^{\prime}$ we have $\left(\mu^{\prime}, M_{i}^{*}\right) \leqslant 0$, which is a contradiction. Thus we have $\left(\tau^{\prime} h^{\prime}, \mu^{\prime}\right)<1$. It follows that $\tau^{\prime} h^{\prime}$ is also an element of $S^{\prime}$. q.e.d.

THEOREM 3. Let $\mathscr{F}=\pi^{\prime} F \subset \mathscr{F}\left(\mathfrak{f}^{\prime}\right)$, that is, $\mathscr{F}$ is the image of $\bar{S}^{\prime} \cap Z$ by the injection $\pi^{\prime} \xi^{\prime-1}: \bar{S}^{\prime} \cap Z_{*}^{\prime} \longrightarrow$ Aut $\left(\Pi^{* *}\right)$, and let $Z^{\prime \prime}$ be the free abelian group defined in Lemma 2. Then

$$
\pi_{1}(G) \cong Z^{\prime \prime} \times \mathscr{F}
$$

If $G$ has no center, then the rank of $Z^{\prime \prime}=Z_{*}^{\prime \prime}$ is the same as the dimension of the center of the maximal compact subgroup $K$ of $G$. The set $2 \pi \sqrt{-1}\left(\bar{S}^{\prime} \cap Z_{*}\right)$ is a complete set of representatives of the torsion part of the center $C$ of the universal covering group of $G$.

Proof. $\pi_{1}(G)$ is isomorphic with $Z^{\prime \prime} \times F$ by Theorem 1 and Lemma 3, 2) and $F$ is isomorphic with $\mathscr{F}$ by Theorem 2. It follows that $\pi_{1}(G)$ is isomorphic with $Z^{\prime \prime} \times \mathscr{F}$. The second statement follows from Lemma 3, 1). The last follows from Lemma 2,2).

q.e.d.

\section{§ 2. Center of a simply connected simple group}

Let $g_{u}$ be a compact simple Lie algebra.

(A) Let $\mathfrak{h}_{u}$ be a Cartan subalgebra of $\mathfrak{g}_{u}$. Then the complexification $\mathfrak{h}^{c}$ of $\mathfrak{h}_{u}$ is a Cartan subalgebra of the complexification $\mathfrak{g}^{c}$ of $\mathfrak{g}_{u}$. The real part $\mathfrak{h}_{0}$ of $\mathfrak{h}^{c}$ is identified with the dual space of $\mathfrak{h}_{0}$ as in Section 1 by means of the Killing form (, ) of $\mathrm{g} c$, so that the root system $\Delta$ of $\mathrm{g} c$ with respect to $\mathfrak{h}^{C}$ is a subset of $\mathfrak{h}_{0}$. Choose a set $\left\{e_{\alpha} ; \alpha \in \Delta\right\}$ of root vectors 
of $\mathrm{g}^{C}$ with respect to $\mathfrak{h}^{C}$ such that $\left[e_{\alpha}, e_{-\alpha}\right]=-\alpha(\alpha \in \Delta)$ and $\left[e_{\alpha}, e_{\beta}\right]=N_{\alpha, \beta} e_{\alpha+\beta}$ $(\alpha, \beta, \alpha+\beta \in \Delta)$ where $N_{\alpha, \beta} \neq 0, N_{\alpha, \beta} \in \boldsymbol{R}$. Let $\Pi=\left\{\alpha_{1}, \cdots, \alpha_{l}\right\}$ be a fundamental system of $\Delta$ and $>$ the lexicographic order of $\Delta$ associated with $\Pi$. Let $\Lambda_{i}^{*} \in \mathfrak{h}_{0}(1 \leqslant i \leqslant l)$ be the dual basis of $\Pi$, that is, $\left\langle\Lambda_{i}^{*}, \alpha_{j}\right)=\delta_{i j}(1 \leqslant i$, $j \leqslant l$ ) and put $\Lambda_{J}^{*}=0, \alpha_{0}=-\mu$, where $\mu$ is the highest root of $\Delta$. Take an involutive trasformation $\rho$ of $\mathfrak{h}_{0}$ with $\rho \Delta=\Delta$ and $\rho \Pi=\Pi$, and put

$$
\mathfrak{h}_{+}=\left\{h \in \mathfrak{h}_{0} ; \rho h=h\right\}
$$

Changing indices of the $\alpha_{i}$ if necessary, we may assume that $\rho \alpha_{i}=\alpha_{i}(1 \leqslant i \leqslant p)$, $\rho \alpha_{p+i}=\alpha_{l_{0}+i}\left(1 \leqslant i \leqslant l_{0}-p\right)$ and $\rho \alpha_{l_{0}+i}=\alpha_{p+i}\left(1 \leqslant i \leqslant l_{0}-p\right)$. Then we have $\Lambda_{i}^{*} \in \mathfrak{h}_{+}$if $0 \leqslant i \leqslant p$. Let $\theta_{\rho}$ be the involutive automorphism of $\mathfrak{g}_{u}$ leaving $\mathfrak{h}_{u}$ invariant, which is characterized by property that its $\boldsymbol{C}$-linear extension $\theta_{\rho}$ to $g^{\boldsymbol{C}}$ satisfies $\theta_{\rho}=\rho$ on $\mathfrak{h}_{0}$ and $\theta_{\rho} e_{\alpha_{2}}=e_{\rho \alpha_{2}}$ for any root $\alpha_{i}$ of $\Pi$. Let $\bar{\alpha}$ denote the image of a root $\alpha$ of $\Delta$ by the orthogonal projection of $\mathfrak{h}_{0}$ onto $\mathfrak{G}_{+}$. Then

$$
\Delta_{0}=\{\bar{\alpha} ; \alpha \in \Delta\}
$$

is the root system of a complex simple Lie algebra of rank $l_{0}$ and

$$
\Pi_{0}=\left\{\bar{\alpha}_{i} ; \alpha_{i} \in \Pi\right\}=\left\{\alpha_{1}, \cdots, \alpha_{p}, \bar{\alpha}_{p+1}, \cdots, \bar{\alpha}_{l_{0}}\right\}
$$

is a fundamental system of $\Delta_{0}$ (Murakami [6], p. 301, p. 302). The lexicographic order $>$ of $\Delta_{0}$ associated with $\Pi_{0}$ is nothing but the one induced by the order $>$ of $\Delta$. Let $\mu_{0}=n_{1} \alpha_{1}+\cdots+n_{p} \alpha_{p}+n_{p+1} \bar{\alpha}_{p+1}+\cdots+n_{l_{0}} \bar{\alpha}_{l_{0}}$ be the highest root of $\Delta_{0}$ and put $n_{0}=1$. Then

$$
\theta=\theta_{\rho} \exp \pi \sqrt{-1} \text { ad } \Lambda_{i_{0}}^{*} \quad\left(0 \leqslant i_{0} \leqslant p, n_{i_{0}}=1 \text { or } 2\right)
$$

is an involutive automorphism of $g_{u}$. We put

$$
\begin{aligned}
& \mathfrak{f}=\left\{x \in \mathfrak{g}_{u} ; \theta x=x\right\}, \mathfrak{p}_{u}=\left\{x \in \mathfrak{g}_{u} ; \theta x=-x\right\}, \\
& \mathfrak{g}=\mathfrak{f}+\sqrt{-1} \mathfrak{p}_{u} .
\end{aligned}
$$

Then $g$ is a real simple Lie algebra, which is a real form of $g c$, and $\mathfrak{t}$ a maximal compact subalgebra of $\mathfrak{g}$. Let $\mathfrak{h}^{\prime}=\mathfrak{h}_{+} \cap \sqrt{-1} \mathfrak{l}^{\prime}$, where $\mathfrak{f}^{\prime}$ is the derived algebra of $\mathfrak{x}$, and $\mathfrak{c}$ the orthogonal complement of $\mathfrak{h}^{\prime}$ in $\mathfrak{h}_{+}$. Then $\mathfrak{h}_{+}, \mathfrak{h}^{\prime}$ and $\mathfrak{c}$ play the same roles as those in Section 1 . So we shall use the same notation as there. 
(B) Let $g$ be the scalor restriction to $\boldsymbol{R}$ of the complexification $\left(\mathfrak{g}_{u}\right) \boldsymbol{c}$ of $g_{u}$. Then $g$ is a real simple Lie algebra, whose maximal compact subalgebra is isomorphic with $g_{u}$.

Theorem. (Murakami [6], p. 295, p. 303) Any real simple Lie algebra $\mathrm{g}$ is obtained from a compact simple Lie algebra $\mathfrak{g}_{u}$ by the construction $(A)$ or $(B)$. In case $(A)$, a fundamental system $\Pi^{\prime}$ of the root system $\Delta^{\prime}$ of $\mathfrak{l}^{\prime} \boldsymbol{C}$ with respect to $\mathfrak{h}^{\prime} \boldsymbol{C}$ and $\mathfrak{c}$ are obtained as follows.

$$
\begin{aligned}
& \text { 1) } \rho=1, i_{0}=0 \quad \Pi^{\prime}=\Pi=\left\{\alpha_{1}, \cdots, \alpha_{l}\right\}, \mathfrak{c}=\{0\} \text {. } \\
& \text { 2) } \rho=1,1 \leqslant i_{0} \leqslant l, n_{i_{0}}=2 \quad \Pi^{\prime}=\left(\Pi-\left\{\alpha_{i_{0}}\right\}\right) \cup\left\{\alpha_{0}\right\}, \mathfrak{c}=\{0\} . \\
& \text { 3) } \rho=1,1 \leqslant i_{0} \leqslant l, n_{i_{0}}=1 \quad \Pi^{\prime}=\Pi-\left\{\alpha_{i_{0}}\right\}, \mathfrak{c}=\boldsymbol{R} \Lambda_{i_{0}}^{*} \text {. } \\
& \text { 4) } \rho \neq 1, i_{0}=0 \quad \Pi^{\prime}=\Pi_{0}, \mathfrak{c}=\{0\} \text {. } \\
& \text { 5) } \rho \neq 1,1 \leqslant i_{0} \leqslant p, n_{i_{0}}=1 \text { or } 2 \quad \Pi^{\prime}=\left(\Pi_{0}-\left\{\alpha_{i_{0}}\{) \cup\{\bar{\xi}\}, \mathfrak{c}=\{0\}\right. \text {, }\right. \\
& \text { where } \xi=\alpha_{i_{0}}+\alpha_{i_{1}}+\alpha_{i_{2}}+\cdots+\alpha_{i_{t}}+\alpha_{k} \text {, } \\
& 1 \leqslant i_{1}, \cdots, i_{t} \leqslant p, p+1 \leqslant k \leqslant l_{0} \text {, } \\
& \left(\alpha_{i_{0}}, \alpha_{i_{1}}\right),\left(\alpha_{i_{1}}, \alpha_{i_{2}}\right), \cdots,\left(\alpha_{i_{t-1}}, \alpha_{i_{t}}\right),\left(\alpha_{i_{t}}, \alpha_{k}\right) \text { are all negative. }
\end{aligned}
$$

Now we want to calculate the center $C$ of the simply connected Lie group with the Lie algebra $g$ constructed in (A) or (B). The center $C$ is isomorphic with the fundamental group $\pi_{1}(G)$ of the adjoint group $G$ of $\mathfrak{g}$. In case $(\mathrm{B})$, the problem is reduced to the one in case (A), 1), since $\pi_{1}(G)$ $\cong \pi_{1}\left(G_{u}\right)$ where $G_{u}$ is the adjoint group of $g_{u}$. In case (A), 1), we have $\pi_{1}(G)=\pi_{1}\left(G_{u}\right) \cong \mathscr{F}\left(\mathfrak{g}_{u}\right)$, which can be calculated by Theorem 2 . So we shall restrict ourselves to find $\bar{S}^{\prime} \cap Z_{*}$ in cases $\left.(\mathrm{A}), 2\right) \sim 5$ ) and a generator of the free part of $\pi_{1}(G)$ in case $\left.(\mathrm{A}), 3\right)$. Let $\mathfrak{l}^{\prime}=\sum_{k=1}^{r} \mathfrak{l}_{k}^{\prime}$ be the decomposition of $\mathfrak{l}^{\prime}$ into simple factors and $\Pi^{\prime *}=\bigcup_{k=1}^{r} \Pi_{k}^{\prime *}$ and $\bar{S}^{\prime} \cap Z_{*}^{\prime}=\prod_{k=1}^{r} \bar{S}_{k}^{\prime} \cap\left(Z_{*}^{\prime}\right)_{k}$ be the corresponding decompositions. We can associate to any element $\gamma$ of $\Pi^{\prime *}$ a positive integer $m_{r}$ and an element $M_{r}^{*}$ of $\mathfrak{h}^{\prime}$ as in Theorem 2: If $r \in \Pi_{k}^{\prime}$, then $m_{r}$ is the coefficient of $\gamma$ in the expression of the highest root of $\Pi_{k}^{\prime}$ as the linear combination of fundamental roots. $\left\{M_{r}^{*} ; \gamma \in \Pi^{\prime}\right\} \subset \mathfrak{h}^{\prime}$ is the dual basis of $\Pi^{\prime}$. If $r \in \Pi^{*}-\Pi^{\prime}$, then $m_{r}=1$ and $M_{r}^{*}=0$. Then by Theo- 
rem 2 any element $z^{\prime}$ of $\bar{S}^{\prime} \cap Z_{*}^{\prime}$ is of the form $z^{\prime}=\sum_{k=1}^{r} M_{\gamma_{k}}^{*}$, where $M_{\gamma_{k}}^{*}$ is an element of $\bar{S}_{k}^{\prime} \cap\left(Z_{*}^{\prime}\right)_{k}$, that is, $\gamma_{k} \in \Pi_{k}^{* *}$ and $m_{\gamma_{k}}=1$.

Case (A), 2). We have $\mu=\sum_{i=1}^{l} n_{i} \alpha_{i}$ since $\rho=1$. We associate to any element $\gamma$ of $\Pi^{\prime *}$ a non-negative integer $n_{r}^{\prime}$ as follows: $n_{r}^{\prime}=n_{i}$ for $\gamma=\alpha_{i} \in \Pi^{\prime}$ and $n_{r}^{\prime}=0$ for $r \in \Pi^{\prime *}-\Pi^{\prime}$. Let $z^{\prime}=\sum_{k=1}^{r} M_{\gamma_{k}}^{*}$ be an element of $\bar{S}^{\prime} \cap Z_{*}^{\prime}$. Then for $i \neq i_{0}, 1 \leqslant i \leqslant l$, we have $\left(\alpha_{i}, z^{\prime}\right) \in\left(\Pi^{\prime}, Z_{*}^{\prime}\right) \subset Z$ and $\left(\alpha_{i_{0}}, z^{\prime}\right)=$ $\left(-(1 / 2)\left(\alpha_{0}+\sum_{\substack{i \neq i_{0} \\ 1 \leqslant i \leqslant l}} n_{i} \alpha_{i}\right), z^{\prime}\right)=-(1 / 2)\left(\sum_{\gamma \in \Pi^{\prime}} n_{\gamma}^{\prime} \gamma, \sum_{k} M_{\gamma_{k}}^{*}\right)=-(1 / 2) \sum_{k} n_{\gamma_{k}}^{\prime}$. It follows that

$$
\bar{S}^{\prime} \cap Z_{*}=\left\{\sum_{k} M_{r_{k}}^{*} ; m_{r_{k}}=1 \cdot \text { for all } k, \sum_{k} n_{r_{k}}^{\prime} \in 2 \boldsymbol{Z}\right\} .
$$

Case (A), 3). Let $a_{i j}=2\left(\alpha_{i}, \alpha_{j}\right) /\left(\alpha_{j}, \alpha_{j}\right)(1 \leqslant i, j \leqslant l)$ be Cartan integers of $\Pi$ and $\left(b_{i j}\right)$ the inverse of the Cartan matrix $\left(a_{i j}\right)$. We associate to any element $\gamma$ of $\Pi^{\prime *}$ a non-negative real number $\lambda_{\gamma}$ as follows: $\lambda_{\gamma}=b_{i_{0}, i} / b_{i_{0}, i_{0}}$ for $\gamma=\alpha_{i} \in \Pi^{\prime}$ and $\lambda_{r}=0$ for $r \in \Pi^{*}-\Pi^{\prime}$. We shall show first that $\mathfrak{h}^{\prime}-$ component of $\alpha_{i_{0}}$ is $-\sum_{r \in \Pi^{\prime}} \lambda_{r} r$. Let $\alpha_{i_{0}}=\lambda \Lambda_{i_{0}}^{*}+\sum_{\alpha_{i} \in \Pi^{\prime}} \lambda_{i}^{\prime} \alpha_{i}\left(\lambda, \lambda_{i}^{\prime} \in \boldsymbol{R}\right)$. From $1=\left(\alpha_{i_{0}}, \Lambda_{i_{0}}^{*}\right)=\lambda\left(\Lambda_{i_{0}}^{*}, \Lambda_{i_{0}}^{*}\right)$, we have $\lambda=1 /\left(\Lambda_{i_{0}}^{*}, \Lambda_{i_{0}}^{*}\right)$. For $i \neq i_{0}, 1 \leqslant i \leqslant l$, from $0=\left(\alpha_{i_{0}}, \Lambda_{i}^{*}\right)=\lambda\left(\Lambda_{i_{0}}^{*}, \Lambda_{i}^{*}\right)+\lambda_{i}^{\prime}$, we have $\lambda_{i}^{\prime}=-\lambda\left(\Lambda_{i_{0}}^{*}, \Lambda_{\imath}^{*}\right)=-\left(\Lambda_{i_{0}}^{*}, \Lambda_{i}^{*}\right) /\left(\Lambda_{i_{0}}^{*}, \Lambda_{i_{0}}^{*}\right)$. If we put $c_{i j}=c_{j i}=\left(\Lambda_{i}^{*}, \Lambda_{j}^{*}\right)(1 \leqslant i, j \leqslant l)$, we have $\Lambda_{i}^{*}=\sum_{j=1}^{l} c_{i j} \alpha_{j}$ and $\delta_{k i}=\delta_{i k}=\left(\Lambda_{i}^{*}, \alpha_{k}\right)=\sum_{j} c_{i j}\left(\alpha_{j}, \alpha_{k}\right)=\sum_{j}\left(c_{i j}\left(\alpha_{j}, \alpha_{j}\right) / 2\right)\left(2\left(\alpha_{j}, \alpha_{k}\right) /\left(\alpha_{j}, \alpha_{j}\right)\right)=\sum_{j} a_{k j}\left(\left(\alpha_{j}, \alpha_{j}\right) c_{j i} / 2\right)$ $(1 \leqslant i, k \leqslant l)$. It follows that $b_{j i}=\left(\alpha_{j}, \alpha_{j}\right) c_{j i} / 2$ and $c_{i j}=\left(2 /\left(\alpha_{i}, \alpha_{i}\right)\right) b_{i j}$. Hence $\lambda_{i}^{\prime}=-c_{i_{0}, i} / c_{i_{0}, i_{0}}=-b_{i_{0}, i} / b_{i_{0}, i_{0}}=-\lambda_{\alpha_{i}}\left(1 \leqslant i \leqslant l, i \neq i_{0}\right)$, as is desired.

Let $z^{\prime}=\sum_{k} M_{\gamma_{k}}^{*}$ be an element of $\bar{S}^{\prime} \cap Z_{*}^{\prime}$. For $i \neq i_{0}, 1 \leqslant i \leqslant l$, we have $\left(\alpha_{i}, z^{\prime}\right) \in\left(\Pi^{\prime}, Z_{*}^{\prime}\right) \subset \boldsymbol{Z}$ and $\left(\alpha_{i_{0}}, z^{\prime}\right)=\left(-\sum_{r \in \Pi^{\prime}} \lambda_{r} \gamma, \sum_{k} M_{\gamma_{k}}^{*}\right)=-\sum_{k} \lambda_{\gamma_{k}}$. It follows that

$$
\bar{S}^{\prime} \cap Z_{*}=\left\{\sum_{k} M_{r_{k}}^{*} ; m_{r_{k}}=1 \text { for all } k, \sum_{k} \lambda_{r_{k}} \in \boldsymbol{Z}\right\}
$$

Let again $z^{\prime}=\sum_{k} M_{r_{k}}^{*}$ be an element of $\bar{S}^{\prime} \cap Z_{*}^{\prime}$. If we put $z=\lambda^{\prime \prime} \Lambda_{i_{0}}^{*}+z^{\prime}$ $\left(\lambda^{\prime \prime} \in \boldsymbol{R}\right)$, then for $i \neq i_{0}, 1 \leqslant i \leqslant l$, we have $\left(z, \alpha_{i}\right)=\left(z^{\prime}, \alpha_{i}\right) \in\left(Z_{*}^{\prime}, \Pi^{\prime}\right) \subset \boldsymbol{Z}$ and $\left(z, \alpha_{i_{0}}\right)=\lambda^{\prime \prime}-\sum_{k} \lambda_{r_{k}}$. It follows that $z \in \bar{S} \cap Z_{*}$ if and only if $\lambda^{\prime \prime}-\sum_{k} \lambda_{r_{k}} \in \boldsymbol{Z}$. Let

$$
\begin{aligned}
& \lambda_{z^{\prime}}=\operatorname{Min}\left\{\left|\sum_{k} \lambda_{r_{k}}+m\right| ; m \in \boldsymbol{Z}, \sum_{k} \lambda_{\tau_{k}}+m \neq 0\right\}, \\
& \lambda_{0}=\operatorname{Min}_{\boldsymbol{z}^{\prime} \in \bar{S}^{\prime} \cap Z_{*}^{\prime}} \lambda_{z^{\prime}} .
\end{aligned}
$$


Let $\lambda_{0}$ be attained by $z_{0}^{\prime}=\sum_{k} M_{\gamma_{k}^{*}}^{*} \in \bar{S}^{\prime} \cap Z_{*}^{\prime}$, that is, $\lambda_{0}=\sum_{k} \lambda_{\tau_{k}^{0}}+m_{0}$ for some integer $m_{0}$. Let $w_{0}^{\prime}=\pi^{\prime} \xi^{\prime-1}\left(z_{0}^{\prime}\right)$ and $w_{0}$ the trivial extension of $w_{0}^{\prime}$ to $\mathfrak{h}_{+}$. Then by Lemma $3 \quad z_{0}=\lambda_{0} \Lambda_{i 0}^{*}+z_{0}^{\prime}$ gives a representative of a generator of the free part of $C$ by multiplying $2 \pi \sqrt{-1}$ and $\tau_{0}=t\left(z_{0}\right) w_{0}$ is a generator of the free part of $\tilde{W}_{*}(S) \cong \pi_{1}(G)$.

Case (A), 4). Since $\Pi^{\prime}=\Pi_{0}$, we have

$$
\bar{S}^{\prime} \cap Z_{*}=\bar{S}^{\prime} \cap Z_{*}^{\prime} \text { and } \mathscr{F}=\mathscr{F}(\mathfrak{k}) \text {. }
$$

Case (A), 5). Let $z^{\prime}$ be an element of $\bar{S}^{\prime} \cap Z_{*}^{\prime}$. For $i \neq i_{0}, 1 \leqslant i \leqslant l$, we have $\left(\alpha_{i}, z^{\prime}\right)=\left(\bar{\alpha}_{i}, z^{\prime}\right) \in\left(\Pi^{\prime}, Z_{*}^{\prime}\right) \subset Z$ and $\left(\alpha_{i_{0}}, z^{\prime}\right)=\left(\xi-\alpha_{i_{1}}-\cdots-\alpha_{i_{t}}-\alpha_{k}, z^{\prime}\right)=$ $\left(\bar{\xi}-\alpha_{i_{1}}-\cdots-\alpha_{i_{t}}-\bar{\alpha}_{k}, z^{\prime}\right)$ is contained in the subgroup of $\boldsymbol{Z}$ generated by $\left(\Pi^{\prime}, Z_{*}^{\prime}\right)$. It follows again that

$$
\bar{S}^{\prime} \cap Z_{*}=\bar{S}^{\prime} \cap Z_{*}^{\prime} \text { and } \mathscr{F}=\mathscr{F}(\mathfrak{k}) \text {. }
$$

Example of Case (A), 3).

$\mathfrak{g}_{u}=A_{l} \quad(l \geqslant 1)$.

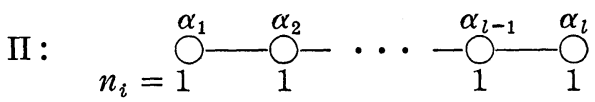

Let $i_{0}=m, \quad 1 \leqslant m \leqslant(l+1) / 2$ and put $n=l+1-m$. Then $b_{m, i}=$ $i n /(m+n) \quad(1 \leqslant i \leqslant m)$ and $b_{m, m+i}=(n-i) m /(m+n)(1 \leqslant i \leqslant n-1)$.
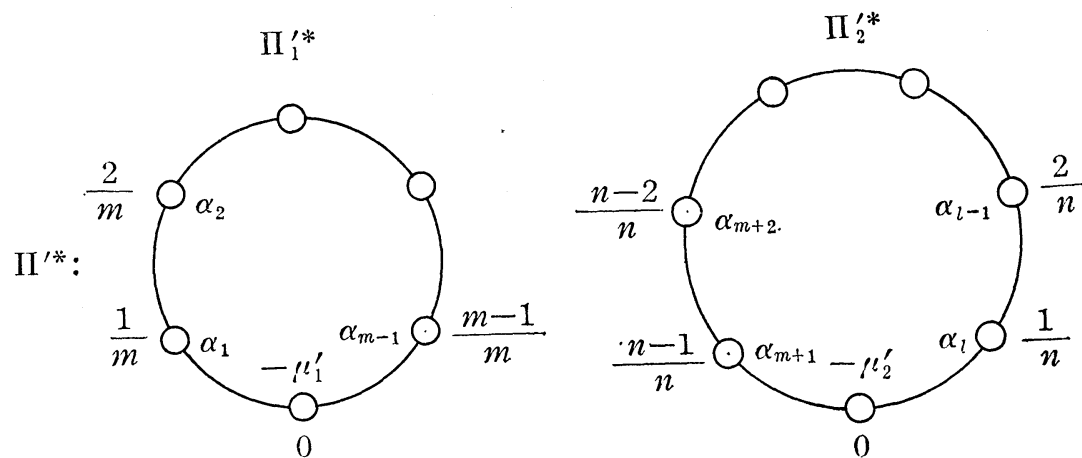

We wrote the number $\lambda_{r}$ at the vertex $\gamma . \quad m_{r}=1$ for all root $\gamma$ of $\Pi^{\prime *}$. Let $\left\{M_{i}^{*} ; 1 \leqslant i \leqslant l, i \neq m\right\} \subset \mathfrak{h}^{\prime}$ be the dual basis of $\left\{\alpha_{i} ; 1 \leqslant i \leqslant l, i \neq m\right\}$ and put $M_{0}^{*}=M_{m}^{*}=0$. Then $\bar{S}^{\prime} \cap Z_{*}^{\prime}=\left\{M_{i}^{*}+M_{m+j}^{*} ; 0 \leqslant i \leqslant m-1,0 \leqslant j \leqslant n-1\right\}$. It follows that $\mathscr{F}\left(\mathfrak{f}^{\prime}\right)$ is the direct product of the groups of "rotations" of 
$\Pi_{1}^{\prime *}$ and $\Pi_{2}^{\prime *}$ so that $\mathscr{F}\left(\mathfrak{f}^{\prime}\right) \cong \boldsymbol{Z}_{m} \times \boldsymbol{Z}_{n}$. Let $d=(m, n)$ and $a$ and $b$ the integers such that $0 \leqslant a \leqslant m-1,0 \leqslant b \leqslant n-1$ and $a n+b m \equiv d(\bmod m n)$. Put $p=m / d$ and $q=n / d$. Then we have

$$
\bar{S}^{\prime} \cap Z_{*}=\left\{M_{p k}^{*}+M_{m+q k}^{*} ; 0 \leqslant k \leqslant d-1\right\}
$$

so that $\mathscr{F} \cong \boldsymbol{Z}_{d}$. We have $\lambda_{0}=d / m n$ so that

$$
z_{0}=(d / m n) \Lambda_{m}^{*}+M_{a}^{*}+M_{m+n-b}^{*}
$$

gives a representative of a generator of the free part of $C$ by multiplying $2 \pi \sqrt{-1}$.

\section{REFERENCES}

[1] M. Goto and E. Kobayashi: On the subgroups of the centers of simply connected simple Lie groupsclassification of simple Lie groups in the large, to appear.

[2] S. Helgason: Differential geometry and symmetric spaces, New York, 1962.

[ 3 ] N. Iwahori and H. Matsumoto: On some Bruhat decomposition and the structure of the Hecke rings of $\mathfrak{p}$-adic Chevalley groups, Inst. des Hautes Etudes Sci. Pub. Math. 25(1965), 237-280.

[4] B. Kostant: Lie algebra cohomology and the generalized Borel-Weil theorem, Ann. of Math. 74 (1961), 329-387.

[5] S. Murakami: On the automorphisms of a real semi-simple Lie algebra, J. Math. Soc. Japan 4 (1952), 103-133.

[6] - Sur la classification des algèbres de Lie réelles et simples, Osaka J. Math. 2 (1965), 291-307.

[ 7 ] A.I. Sirota and A.S. Solodovnikov: Non-compact semi-simple Lie groups, Uspekhi Mat. Nauk 18, No. 3 (1963), 87-144. English translation of the same issue, 85-140.

[8] M. Takeuchi: On the fundamental group and the group of isometries of a symmetric space, J. Fac. Sci. Univ. Tokyo, I, 10(1964), 88-123.

Osaka University 